Bull. Soc. Chim. Belg., 61, pp. 181-202, 7 fig., 1952

\title{
VII. - Analyse chimique et électrotitrimétrique de l'acide ribonucléique de la levure
}

\author{
par \\ J.M. GHUYSEN et V. DESREUX (Iiège)
}

Summars. -- The fractions of different molecular weights of yeast ribonucleic acid have been analyzed by chromatography and by the electrotitration curves. The results obtained by these two methods are practically the same if' we assume that therc is 0,65 secondary phosphoric acid group per four purine and pyrimidine bases. The chemical composition of all the fractions obtained by fractional-extraction, is practically the same, so that there must be a statistical distribution of the bases in the high molecular weight ribonucleic acid. The depolymerisation to very low molecular weight degradation products does not seem to liberate free secondary phosphoric acid groups.

It appears that in a "native " yeast ribonucleic acid, the molecular proportions of the four bases are nearly identical.

\section{I. - INTRODLCTION}

Nous avons montré que l'acide pentosenucléique de la levure extrait par le procédé à la soude se caractérise par une hétérogénéité continue; d'autre part, les précipités de cet acide présentent les caractéristiques des solutions solides ( $\left.{ }^{1}\right)$

Par extraction fractionnée effectuée en système discontinu à $\mathrm{pH} 4,5$, nous avons obtenu une série de fractions dont les poids moléculaires variaient de 7.000 à 46.500 ( ${ }^{2}$ ); l'hétérogénéité de l'acide ribonucléique dépend certainement de la masse.

Nous avons recherché par analyse chromatographique

(1) J.M. Gitcysen : Bull. Soc. Chim. Belg., 59, 490 (1950).

( $\left.{ }^{2}\right)$ V. Desreux et J.M. Girursen: Bull. Soc. Chim. Belg. 60, 410 (1951). 
et électrotitrimétrique si, à cette hétérogénéité de grandeur moléculaire, correspondait une hétérngénéité de composition chimique.

Nous avons enfin recherché quelle était l'incidence des résultats obtenus sur les conceptions actuelles relatives à la structure de l'acide pentosenucléique de la levure.

\section{II. - INALYSE CHROMATOGRaphIQUN}

\section{A) Parlie expérimenlale}

\section{a) Matériel utilisé}

Nous rappelons que les fractions obtenues par extraction fractionnée à pH 5,5 sont désignées par le rapport : volume du précipitant

volume solvant + volume précipitant

ou $\gamma$ auquel chaque fraction a été extraite.

Les fractions de $\gamma$ décroissants $(\gamma: 1,0 \rightarrow 0,0)$ se caractérisent done par une solubilité de plus en plus faible et par un poids moléculaire de plus en plus élevé $(7.500 \rightarrow 38.000)$; à partir de la fraction $\gamma: 0,0$, repurifiée par la même méthode, nous avons obtenu l'acide $\gamma: 0,0$, de poids moléculaire 46.500 .

Nous avons également étudié trois fractions préparées a partir d'acide ribonucléique brut, par précipitation fractionnée au moyen de deux systèmes solvant-précipitant d'un $\mathrm{pH}$ inférieur à 5.5 , L'emploi du précipitant de Chantrenne $\left({ }^{3}\right.$ ) (acide acétique : deux parties; acétone : une partie; eau : deux parties) conduit à l'obtention d'une fraction $A$ très peu soluble dans ce milieu et une fraction $B$ très soluble et l'emploi du précipitant : $\mathrm{CH}_{3} \mathrm{OH}=20 \% ; \mu=1,7$ ( NaCl) et pH 3,5 (acétate : $\mu=0,10)$ donne une fraction insoluble $A_{1}^{\prime}\left({ }^{1}\right)$. L'obtention de ces fractions a été décrite dans nos publications antérieures.

\section{b) Technique chromatographique}

Chaque fraction est séchée à $100^{\circ} \mathrm{C}$ pendant douze heures. Lanalyse chromatographique est ensuite effectuée sur chacune des fractions suivant la méthode décrite par Markham et Smith $\left(^{4}\right.$ ). Nous avons toutefois adopté cortaines modifications de cette technique introduites au laboratoire de J. Brachet (communication de $H$. Chantrenne), particulièrement en ce qui concerne le séchage du chromatogramme et l'extraction des bases puriques et pyrimidiques.

(3) H. Chantrenne : Bull. Soc. Chim. Belg., 55-5) (1946).

(4) R. МанкHAM et J.D. SмiтH : Biochem. J. 45-294 (1949); 46-509 (1950); 49-401 (1951). 
L'acide nucléique ( 10 à $20 \mathrm{mg}$ par cc) est hydrolysé quantitativement par $\mathrm{HCl} \mathrm{N}$. en bases puriques et pyrimidiques à $100^{\circ} \mathrm{C}$ et en tube scelle. Les produits d'hydrolyse sont ensuite séparés par chromatographie sur papier Whatman $\mathrm{N}^{\circ} 1$ ou $\mathrm{N}^{\circ} 4$ à l'aide d'une solution chlorhydrique de butanol tertiaire. Après séparation, séchage et traitement à l'ammoniaque, on localise les taches en illuminant le papier par la lumière $\mathbf{U}$. V. émise par une lampe HP Philips dont l'enveloppe extérieure de verre a été éliminée. Cette lumière est filtrée par passage au travers d'une solution contenant $235 \mathrm{grs}$ de $\mathrm{NiSO}_{4} \cdot 7 \mathrm{H}_{2} \mathrm{O}$ et $67,1 \mathrm{grs}$ de $\mathrm{CuSO}_{4}$. $\boldsymbol{7} \mathrm{H}_{2} \mathrm{O}$ par litre (épaisseur $7 \mathrm{cms}$. : Bowen $\left({ }^{5}\right)$ ). Les bases extraites par HCI 0.1 N. sont enfin dosées spectrophotométriquement comme décrit par Markham et Smith. Les densités optiques utilisées pour nos calculs sont celles proposées par ces deux auteurs en septembre 1951.

c) Dosage d'azote et de phosphore

La minéralisation a été faite sur des prises d'essai contenant 50 à $500 \gamma$ de phosphore et 100 à $1200 \gamma$ d'azote à l'aide d'acide sulfurique concentré (un $\mathrm{cc}$ ) et de sulfate de potasse (un gr.). Le dosage du phosphore a été effectué colorimétriquement à l'aide du réactif molybdique et celui de l'azote à l'aide du réactif de Nessler.

Ces dosages sont pratiqués sur deux prises d'essai de la môme fraction à partir desquelles on effectue quatre déterminations séparées de chaque élément.

\section{B) Résultats obtenus}

a) Fractions obtenues par extraction fractionnée à $p H \quad 5,5$

Dans le tableau I nous avons consigné les résultats analytiques obtenus exclusivement par chromatographie indépendamment de tout dosage d'azote ou de phosphore. Les rapports molaires des bases puriques et pyrimidiques des différentes fractions isolées par extraction fractionnée ont été calculés en prenant comme somme de ces bases une valeur arbitraire égale à 4 .

Ce calcul n'implique aucune hypothèse sur la structure de l'acide nucléique.

Une déphosphorylation partielle ainsi qu'une contamination par dérivés protéiques étant toujours à craindre, nous avons préféré discuter essentiellement nos résultats sur une base purement comparative.

(5) E.J. Bowen : J. Chem. Soc. 2236 (1932); 76 (1935). 
TABLEAU I

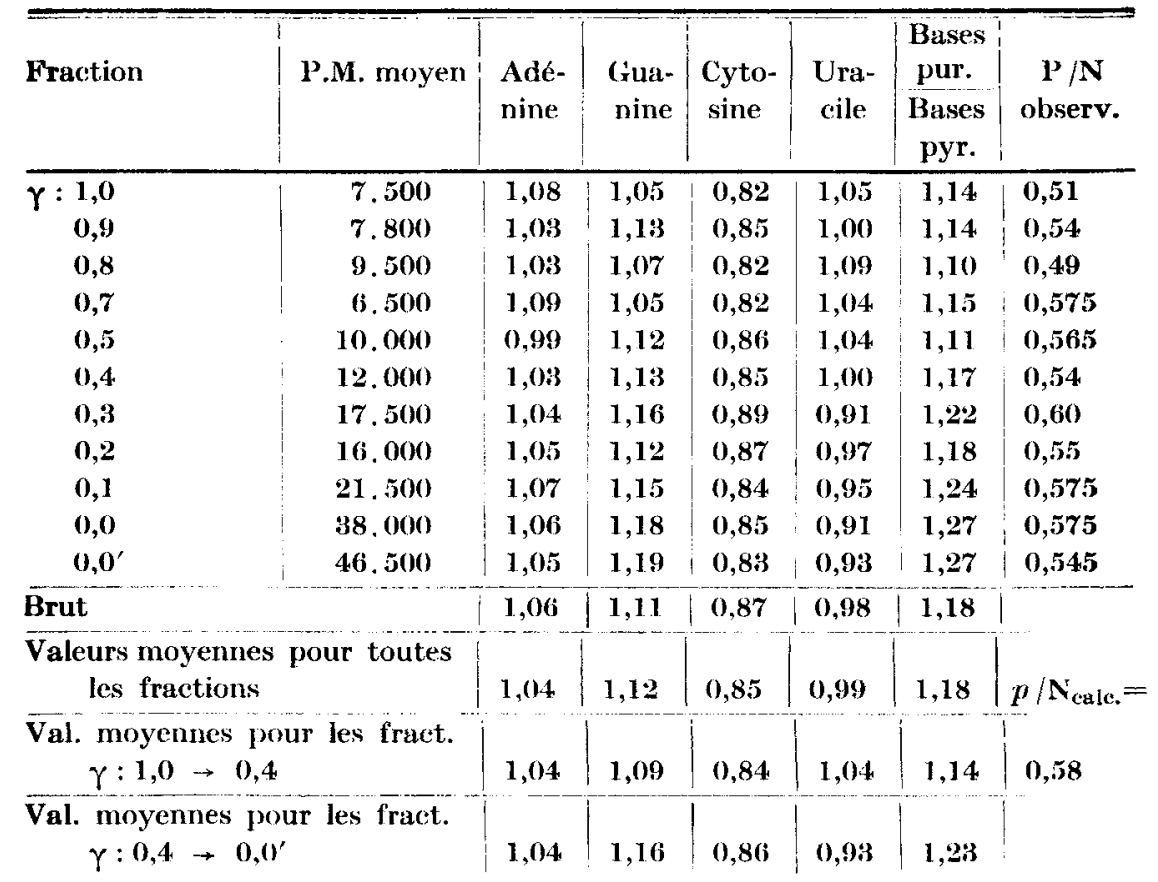

L'examen des chiffres de ce tableau révèle que toutes les fractions extraites depuis $\gamma: 1,0$ jusqu'à $\gamma: 0,0$ et isolées de l'extrait par précipitation à l'aide de l'alcool, ont approximativement la même composition en bases puriques et pyrimidiques quel que soit le poids moléculaire. De mème la fraction $\gamma: 0,0^{\prime}$ a une composition identique à celle de la fraction $\gamma: 0,0$ d'où la première a été isolée qprès élimination par extraction fractionnée d'environ $60 \%$ de produit.

Magasanik \& Chargafi $\left({ }^{6}\right)$ en conformité avec ce qui précède, ont trouvé que le dialysat et le résidu de la dialyse d'un acide nucléique brut de levure avaient pratiquement la même composition en bases puriques et pyrimidiques.

Il semble toutefois que nos fractions les moins dépolymérisées $\left(\gamma: 0,4 \rightarrow 0,0^{\prime}\right)$ sont légèrement plus riches en bases puriques qu'en bases pyrimidiques, le rapport des bases puriques aux bases pyrimidiques étant de 1,14 pour les fractions $\gamma: 1 \rightarrow 0,4$ et de 1,23 pour les fractions $\gamma: 0,4 \rightarrow 0,0^{\prime}$;

$\left({ }^{6}\right)$ S. Magasanik et E. Chargaff : Biochim. Biophys. Acta 7-396 (1951). 
cet enrichissement est dù à un pourcentage inférieur en uracile et à un pourcentage supérieır en guanine. D'après des résultats obtenus par différents auteurs, entre autres par Magasanik et Chargaff $\left({ }^{6}\right)$, Backer et Allen ( $\left.{ }^{7}\right)$, la dégradation de l'acide nucléique par la soude se lerait avec élimination préférentielle d'acide uridylique, l'acide adénylique s'éliminant en dernier lieu. L. acide uridylique se retrouverait donc au cours du fractionnement dans les fractions les plus solubles $(\gamma: 1,0 \rightarrow 0,4)$, la majeure partie de ce mononucléotide étant cependant éliminée au cours de la précipitation alcoolique de l'extrait. 1)'autre part, l'acide guanylique représenterait environ les deux tiers des bases constitutives du résidu non dialysable obtenu par action de la ribonucléase sur l'acide nucléique. le pourcentage en guanine des fractions de poids moléculaires jevés, quelque peu supérieur à celui trouvé pour les fractions de bas poids moléculaires, pourrait donc être dû à une légère dégradation enzymatique se produisant au cours du premier stade d'isolement de l'acide par la soude c.à.d. lorsque le contenu celhulaire n'est pas encore au $\mathrm{pH}$ très alcalin, où le ferment est inactivé.

Nous ne pouvons évidemment pas éliminer la possibilité d'une certaine dégradation au cours du fractionnement à pH 5,j et par conséquent contamination de la fraction extraite par les éventuels produits de dégradation très solubles de l’acide nucléique restant dans la colonne à extraction. Cette dégradation, dans nos conditions expérimentales de fractionnement, doit cependant ĉtre très faible car la composition moyenne des diverses fractions est identique à celle du produit de départ et chaque raction a eté isolée de l'extrait par précipitation alcoolique; au cours de cette dernière manipulation, on doit donc éliminer la majeure partie de produit de degradation très solubles eventuellement formés, ce qui entraînerait une modification des proportions des bases. Ces résultats montrent egalement que toute la ribonucléase doit avoir été détruite lors du traitement at la soude et que l'hétérogénéité trouvée ne peut provenir d'une éventuelle dégradation enzymatique se faisant au cours du fractionnement.

(7) J.E. BACKEK and F'.W. AlLEN : J. Biol. Chem. 183-63:3 (1950). 
b) Fractions obtenues par précipitation fractionnée au $\mathrm{pH}$ de 3,5 $\left(\mathrm{A}^{\prime}{ }_{1}\right)$ ef en présence d'acide acétique $(\mathrm{A}$ at $\mathrm{B})$

Le tableau II résume les données analytiques relatives à ces fractions ef calculées à nouveau en adoptant une somme arbitraire de quatre bases.

\section{TABLEAL I]}

\begin{tabular}{|c|c|c|c|c|c|c|c|}
\hline \multirow[b]{2}{*}{ Fraction } & \multirow{2}{*}{ P. M. moyen } & \multicolumn{2}{|c|}{ 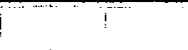 } & ! & \multicolumn{3}{|c|}{ Bases } \\
\hline & & $\begin{array}{l}\text { ade- } \\
\text { luine }\end{array}$ & $\begin{array}{l}\text { gua- } \\
\text { nine }\end{array}$ & $\begin{array}{l}\text { rylo } \\
\text { sine }\end{array}$ & $\begin{array}{l}\text { uraei- } \\
\text { le }\end{array}$ & $\begin{array}{l}\text { pur. } \\
\text { Bases } \\
\text { pyr. }\end{array}$ & $\begin{array}{c}P / N \\
\text { observ. }\end{array}$ \\
\hline $\bar{A}$ & 13.000 & 1.16 & 1.11 & 0,83 & 0,91 & 1,30 & $\overline{0,45}$ \\
\hline B & 4,000 & 0,91 & 0.983 & $C_{0,70}$ & 1,38 & 0.85 & $0,50.5$ \\
\hline $\mathbf{A}_{1}^{\prime}$ & 18.500 & 1,22 & 1,10 & 10,73 & 0,96 & 1,37 & 0,48 \\
\hline Brui & & $1.06 \mathrm{6}$ & 1,11 & 0.86 & 0,98 & 1,18 & \\
\hline
\end{tabular}

les fractions les moims solubles 1 el $\lambda^{\prime}$, contipunent proportionnellement plus diadénine que les echantillons dacide nucléique isolés all pH 5,5 par extraction fractionnés. Le rapport des hases puriques aux hases pyrimidiques est très élevé dans le cas de ces deux échantillons ot trós faible dans le cas de la fraction B. Cette fraction doit en effet contenir les produits de dégradation tres solubles el est tros riche en uracile; ctle conticut proportionnellement beanconp moins d'adénine que les fractions 1 et $N_{1}$.

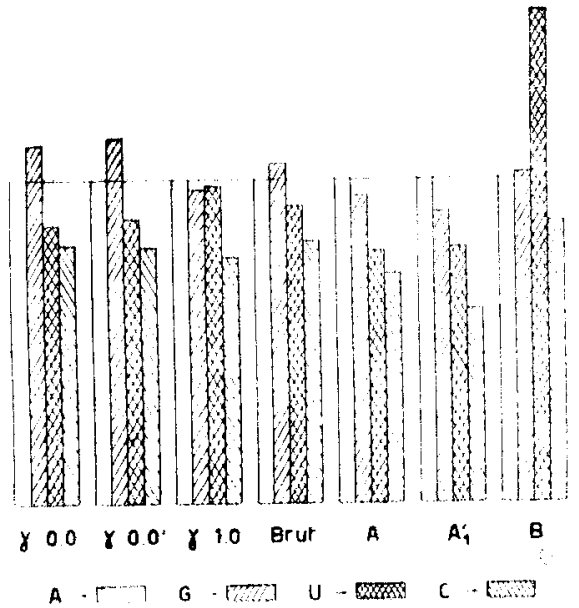

Fig. 1

\section{(i) Discussion}

Dans la figure 1 , moms comparoms la ri. parliliun des bases pour les lmations $y: 1),(1 ; 1), 0^{\prime}$;

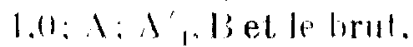
la yountile d'arlenine

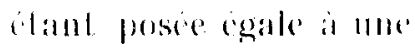
mole ceetle base de eomparaison nous semble logique puisque, lors de la rlégradation alcaline, l'acide adénylique serait le composant le plus 
difficilement éliminable. On voit que la fraction la plus soluble isolée au pH 5,5 $\gamma: 1,0)$ contient proportionnellement beaucoup moins d'acide uridylique que la fraction $B$ très soluble isolée à un $\mathrm{pH}$ plus acide. Ceci est étrange à première vue puisque le fractionnement a été beaucoup plus efficace au $\mathrm{pH} 5,5$ par extraction fractionnée qu'au $\mathrm{pH}$ de 3,5 par précipitation.

Nous avons vu dans un article précédent $\left({ }^{1}\right)$ que l'emploi du précipitant à l'acide acétique de Chantrenne provoquait une légère dégradation se manifestant par une augmentation de solubilité de la fraction A; il en était de même pour tout milieu à $\mathrm{pH}$ trop acide utilisé au cours du fractionnement. D'autre part, l'acide ribonucléique très polymérisé de constante de sédimentation $\mathrm{S}_{20}$ égale à $5,7 \mathrm{~S}$ (fraction $\gamma: 0,0$ ) subit une dépolymérisation à $\mathrm{pH} 3$; ces résultats doivent cependant encore être confirmés systématiquement. C'est ainsi qu'une solution à $0,35 \%$ de cette fraction (en solution $\mathrm{KCl}, \mu$ : 0,166 ), après avoir été amenée à $\mathrm{pH} 3,0$ et ramenée à $\mathrm{pH} 5,5$, se caractérise par une constante de sédimentation de 3,45 mesurée dans les mèmes conditions que précédemment. Il est donc possible qu'en plus de la dégradation alcaline se produisant au cours du traitement de la levure, il y ait une dépolymérisation accompagnée d'une dégradation acide pendant le fractionnement, au $\mathrm{pH}$ de 3,5 , dégradation qui se ferait également avec élimination préférentielle d'acide uridylique.

L'instabilité de l'acide nucléique en milieu acide nous pousse à ne pas considérer la composition des fractions $\mathrm{A}$ et $\mathrm{A}^{\prime}{ }_{1}$ comme très représentative de la répartition des bases dans l'acide ribonucléique "natif"; ces deux fractions ont des compositions analogues entre elles mais assez divergentes de celles des échantillons isolés à pH 5,5.

Nous limiterons donc la discussion de nos résultats analytiques essentiellement aux fractions isolées à ce dernier $\mathrm{pH}$.

a) De l'examen des chiffres du tableau I il résulte apparemment que l'hétérogénéité de l'acide ribonucléique est essentiellement fonction de la grandeur moléculaire et ne dépend que peu de la répartition en bases puriques et pyri- 
midiques. Cette conclusion semble montrer que la dégradation physique (dépolymérisation) se fait sans grande modification de composition en ces bases tout au moins dans les limites des grandeurs moléculaires de nos fractions. Les deux types de dégradation, physique et chimique, seraient donc plus ou moins indépendants bien que concomitants, ce qui confirmerait jusqu'à un certain point, l'hypothèse émise par Magasanik et Chargaff $\left({ }^{6}\right)$ sur la structure des acides ribonucléiques : une chaîne de bases puriques sur laquelle se grefferaient des chaînes latérales constituées essentiellement de bases pyrimidiques. Il faudrait en outre supposer que la répartition des bases, aussi bien dans la chaîne principale que dans les chaînes latérales fut assez régulière.

b) Pouvons-nous prévoir maintenant ce que serait la composition hypothétique en hases d'un acide ribonucléique "natif" de lev'ure?

Comparons nos résultats $\left(\gamma: 1,0 \rightarrow 0,4 ; 0,4 \rightarrow 0,0^{\prime}\right.$ et brut)

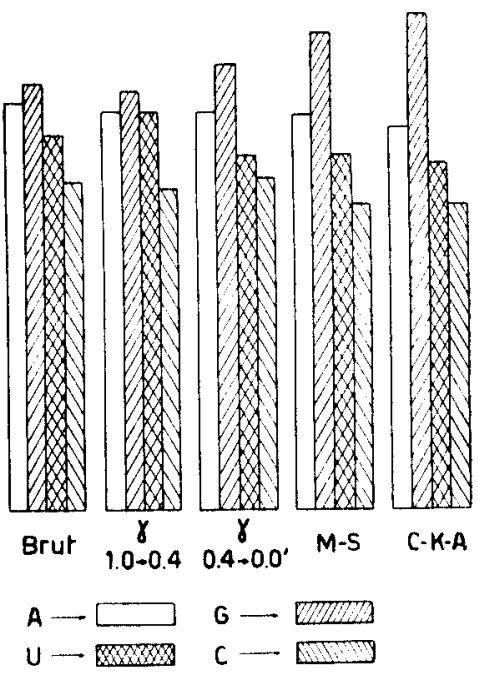

Fig. 2 à ceux obtenus par Markham et Smith $\left({ }^{4}\right)$ et par Cavalieri, Kerr et Angelos $\left({ }^{8}\right)$ (échantillon dialysé), la somme des bases puriques et pyrimidiques étant dans chaque cas posée arbitrairement égale à quatre.

L'aspect général des distributions de tous ces échantillons est asse $z$ semblable (voir graphique 2); la seule différence nette est la proportion de guanine ainsi que le montrent les chiffres du tableau suivant (III).

$\left({ }^{8}\right)$ L.F. Cavaleri, S.E. Kerr et A. Angelos : J.A.C.S., 73, 2567 (1951). 


\begin{tabular}{|c|c|c|c|c|c|}
\hline fractions & guanine & adénine & cytosine & uracile & $\begin{array}{l}\text { bases puriq } \\
\text { bases pyri- } \\
\text { midiques }\end{array}$ \\
\hline brut & 1,11 & 1,06 & 0,86 & 0,98 & 1,18 \\
\hline $1,0 \rightarrow 0,4$ & 1,09 & 1,04 & 0,84 & 1,04 & 1,14 \\
\hline $0,4 \rightarrow 0,0^{\prime}$ & 1,16 & 1,04 & 0,86 & 0,93 & 1,23 \\
\hline $\mathbf{M}-\mathbf{S}$ & 1,24 & 1,03 & 0,80 & 0,94 & 1,31 \\
\hline$C-K-A$ & 1,29 & 1,00 & 0.80 & 0,91 & 1,34 \\
\hline
\end{tabular}

Si les échantillons étudiés étaient totalement débarrassés des produits de dégradation, nous pourrions dire à priori que plus le pourcentage des bases pyrimidiques est élevé et moins le produit est chimiquement dégradé, puisque aussi bien en milieu alcalin que, semble-t-il, en milieu acide et sous l'action de la ribonucléase ces bases pyrimidiques sont éliminées préférentiellement.

Nous n'avons aucune indication sur l'hétérogénéité et la grandeur moléculaire des échantillons de Markham et Smith et de Cavalieri, Kerr et Angelos; remarquons cependant que le pourcentage en guanine de ces produits est assez bien plus élevé que celui de nos fractions ce qui semblerait indiquer une élimination assez importante de bases pyrimidiques (partiellement sous l'action de la ribonucléase par exemple). D'autre part, nos fractions $\gamma: 1,0 \rightarrow 0,4$ doivent certainement contenir des produits de dégradation chimique (pourcentage d'uracile plus élevé.)

Rappelons enfin que le pourcentage supérieur en guanine des fractions de poids moléculaires élevés $\left(\gamma: 0,4 \rightarrow 0,0^{\prime}\right)$ est vraisemblablement dû également à un enrichissement en cette base, suite à une action enzymatique de la ribonucléase au cours du traitement de la levure.

Prenant en considération ce qui précède, nous sommes amenés à supposer que la composition hypothétique de l'acide ribonucléique "natif " doit différer légèrement de celle caractérisant les fractions $\gamma: 0,0$ et $0,0^{\prime}$, le pourcentage en guanine devant ètre plus faible et celui de l'uracile et de la cytosine plus élevé; cette composition statistique tendrait donc vers celle requise par une distribution équimoléculaire des quatre bases $(*)$.

(*) Voir le travail récent de R. 'Tномas, Bioch. Biophys. acta, $8,71,1952$. 


\section{c) Remarque}

Les conclusions précédentes ne peuvent cependant ètre acceptées qu'avec beaucoup de prudence.

Ghuysen (1) a en elfel montré que les précipités d'acide nucléique se comporlaient comme des solutions solides et que ces phénomènes étaient plus manifestes au pH 5,5 qu'au pH 3,5. Il est done possible qu'au pH 3,0-3,5 l'élimination des petiles molécules de polynucléotides on de nucléotides soit, en fait, plus aisée qu'à un pH plus alcalin; l'impossibilité d'obtenir au pH 3,5, des fractions de poids moléculaires très différents el clovés serait alors due à la dépolymérisation se produisant a ce pH acide.

Si loules les fractions d'acide nucléique qui sont, comme nous l'avons vu antérieurement, polydispersées, se comportent comme des solutions solides, la composition en bases de ces fractions doit etre fonction des conditions expérimentales dans lesquelles elles ont été obtenues, puisque la dégradation donne naissance à des produits de décomposition de structure légèrement différente. Il n'est donc pas étonnant, croyons-nous, de trouver dans la littérature des résultats analytiques assez peu concordants et il serait dangereux de tirer d'expériences de dégradation des conclusions trop hàtives sur la structure des acides nucléiques à moins de disposer de fractions aussi honogènes que possible et de degrés de polymérisation différents.

\section{III. - INAI.YSE GHACTROTITRIMÉTRIQUE}

\section{a) Technique}

Les courbes expérimentales d'électrotitration ont été tracées entre pH 3 et pH 11 à l'aide de l'électrode de verre et en milieu $\mathrm{KCl} 0,2 \mathrm{M}$. L'acide nucléique ( séché à froid et sous vide en présence de $\mathrm{P}_{2} \mathrm{O}_{5}$ ) est titré par HCl N. et KOII N.

Nous avons effectué ces d́lectrotitrations en présence d'une quantité assez notable de $\mathrm{KCl}$ afin de minimiser les effets de la variation de force ionique au cours du titrage sur les valeurs des coefficients d'activité et afin de pouvoir négliger l'influence du nucléate sur ces coefficients.

D'autre part, nous avons choisi $\mathrm{KCl}$ et non $\mathrm{NaCl}$ comme d'ailleurs KOH au lieu de NaOH afin de maintenir constantes les caractéristiques de l'électrode de verre jusqu'au pII élevés. 
Liemploi de solutions d'acide ou de bases de titres élevés minimisent également les effets de dilution.

Moyennant ces précautions, nous avons vérifié que les courbes d'électrotitration aller et retour $\mathrm{pH} 7 \rightarrow \mathrm{pH} 3 \rightarrow \mathrm{PH} 11 \rightarrow \mathrm{pH} 3$ d'une fraction coincident à 0,05 équivalents près; on n'observe aucune fixation apparente continue de soude.

L'anomalie signalée précédemment ( $\left.{ }^{*}\right)$ disparaît par conséquent si l'on travaille dans des conditions de parfaite réversibilité de l'électrode de verre et de constance de force ionique.

La conversion des activités de l'ion $\mathrm{H}^{+}$en concentrations a été faite par un titrage à blanc de la solution de $\mathbf{K C l}$.

Les courbes expérimentales couvrant un domaine de $\mathrm{pH}$ de 3,0 à 11,0 , nous avons à considérer comme fonctions titrables en totalité ou partiellement, celles mentionnées dans le tableau IV.

TABLEAU IV. - Valeurs des coefficients d'ionisation à différents $\mathrm{pH}$

\begin{tabular}{|c|c|c|c|c|c|c|}
\hline \multirow[t]{2}{*}{ pH | } & & \multicolumn{2}{|c|}{$\mathrm{NH}_{2}$} & $\mathbf{O H}$ & \multicolumn{2}{|c|}{ |OH énolique (-CO-NH-) } \\
\hline & $\begin{array}{l}\text { guanine } \\
p k: 2,3\end{array}$ & $\begin{array}{c}\text { adénine } \\
p k: \mathbf{3 , 7}\end{array}$ & $\begin{array}{l}\text { cytosine } \\
p k: 4,2\end{array}$ & $\begin{array}{c}\text { phosphori- } \\
\text { que secon- } \\
\text { daire } \\
p k: \quad \mathbf{6 , 0}\end{array}$ & $\begin{array}{l}\text { guanine } \\
p k: 10,1\end{array}$ & $\begin{array}{c}\text { uracile } \\
p k: 10,2\end{array}$ \\
\hline 3 & 0,83 & 0,16 & 0,06 & 0,00 & $\ldots$ & - \\
\hline 4,5 & 0,99 & 0,86 & 0,67 & 0,03 & - & - \\
\hline 6,0 & 1,00 & 0,99 & 0,98 & 0,50 & $\ldots$ & - \\
\hline 7,0 & 1,00 & 1,00 & 1,00 & 0,91 & - & - \\
\hline 8,0 & 1,00 & 1,00 & 1,00 & 0,90 & 0,008 & 0,006 \\
\hline 11 & 1,00 & 1,00 & 1,00 & 1,00 & 0,89 & 0,86 \\
\hline
\end{tabular}

Ces valeurs de $\mathrm{pK}$ sont celles données par Levene $\left({ }^{10}\right)$ pour l'acide nucléique et pour les mononucléotides isolés.

Pour les groupes $\mathrm{NH}_{2}$ et le groupe $\mathrm{OH}$ phosphorique secondaire, Levene a proposé des valeurs concordantes dans les deux cas, mais les $\mathrm{pK}$ des groupes $\mathrm{OH}$ énoliques de la guanine et de l'uracile diffèrent selon que l'on envisage le mononucléotide ou l'acide nucléique. Les valeurs plus élevées de $\mathrm{pK}$ proposées par Levene dans le dernier cas, ont été déduites des courbes d'électrotitration de l'acide nucléique, admettant la structure tétranucléolidique régulière.

(9) I. Delcambe et V. Desreux : Bull. Soc. Chim. Belg., 59-521 (1950).

(10) Levene and Bass : Nucleic acids N.H.. p. 284 (1931). 
Cette hypothèse, dans notre cas, étant assez proche de la réalité, nous avons adopté pour ces groupes $\mathrm{OH}$ de la guanine et de l'uracile, les valeurs de pK : 10,1;10,2.

Les groupes $\mathrm{OH}$ phosphoriques primaires n'étant pas titrables dans les limites du pH adoptées, nous pouvons nous attendre à obtenir le mème type de courbe d'électrotitration pour toutes les fractions obtenues par extraction fractionnée puisque la composition en bases puriques et pyrimidiques de ces fractions est pratiquement la même, à condition que les seuls groupements titrables soient bien ceux mentionnés dans le tableau IV et que le nombre de groupes $O H$ phosphoriques secondaires soit le même dans toutes les fractions. Les courbes d'électrotitration ont été tracées en faisant à nouveau abstraction de tout dosage d'azote et de phosphore. La quantité d'acide nucléique utilisée dans chaque essai n'étant pas la mème et le degré de dessiccation n'étant pas constant, nous avons donc multiplié les équivalents de bases fixées à chaque $\mathrm{pH}$ par un coefficient de valeur telle que la quantité totale de base fixée entre $\mathrm{pH} 3$ et 11 soit la mème dans chaque cas ( 484 équivalents $10^{-6}$ ). Toutes les courbes suivantes n'ont donc qu'une signification comparative.

b) Résullats

Le tableau $\mathrm{V}$ résume les données expérimentales et les

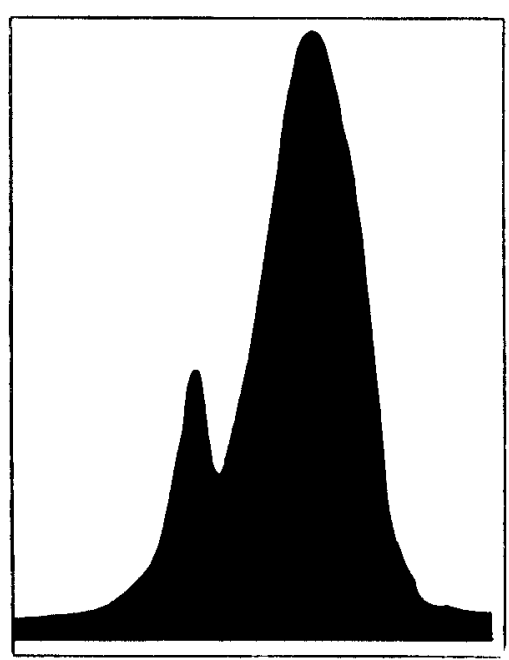

liig. 3 graphiques 1,5 et 6 représentent les courbes de titration, respectivement des fractions $\gamma 0,9 ; 0.8 ; 0,7 ; 0,5$; des iractions $\gamma 0,4 ; 0,3 ; 0,2 ; 0,1$; 0,0 ; et enfin des fractions $\mathrm{A}$; $\Lambda^{\prime}$; et $\mathrm{B}$.

Nous ne disposions plus suffisamment de matière de la fraction $\gamma \quad 0,0^{\prime}$; d'autre part la fraction $\gamma 1,0$ riche en produits de dégradation, semblait être contaminée par une autre substance que l'acide nucléique; une électrophorèse de cette fraction 


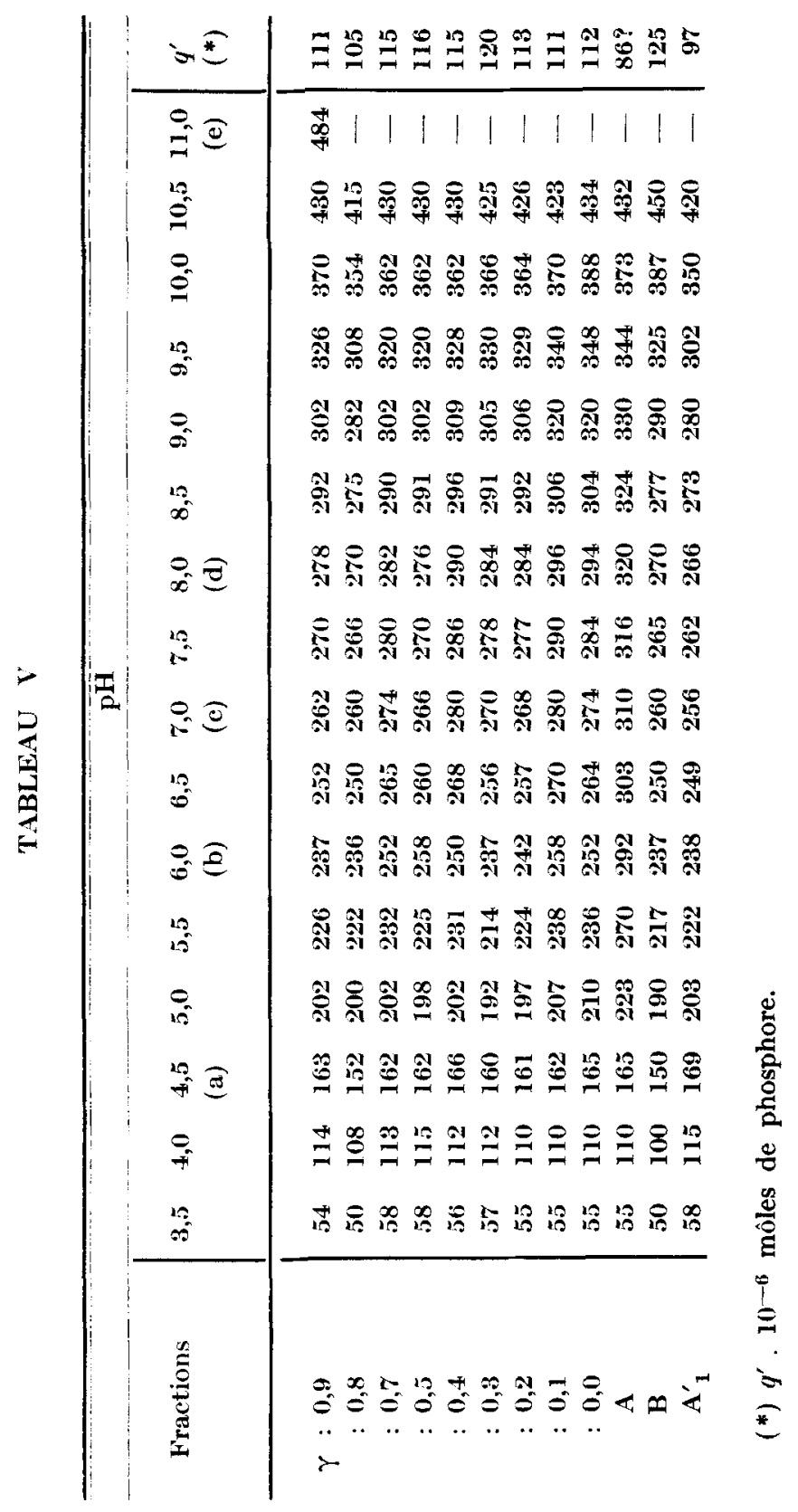




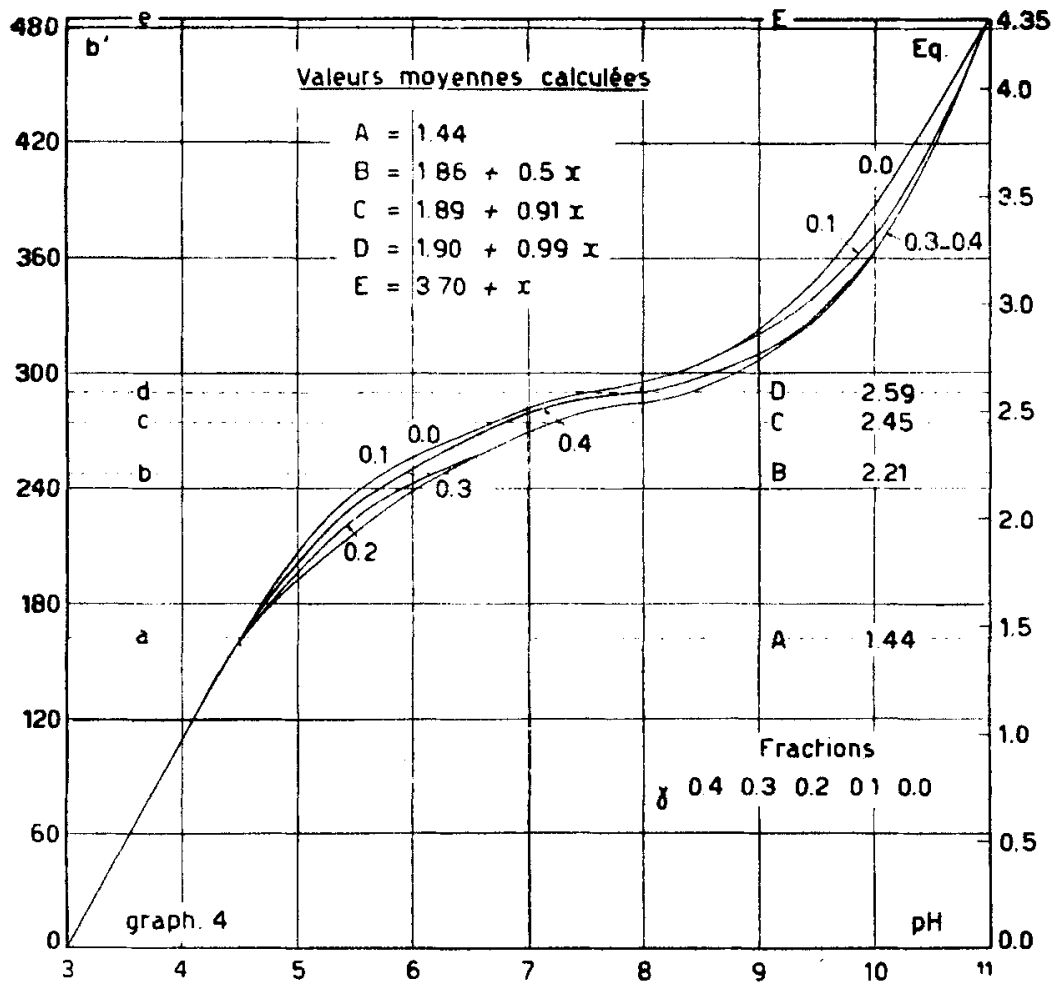

effectuée à $\mathrm{pH} 7$, montre clairement cette hétérogénéité (voir figure 3 ) $\left(^{*}\right)$.

Considérons d'abord les fractions obtenues par extraction fractionnée (graphiques 4 et 5). La dispersion des résultats est très faible de $\mathrm{pH} 3$ à $\mathrm{pH} 4,5$ (domaine de titration du groupe $\mathrm{NH}_{2}$ ) mais elle est plus grande du pH 4,5 au pH 7 (domaine de titration des groupes $\mathrm{NH}_{9}$ et du groupe $\mathrm{OH}$ phosphorique secondaire)et au-dessus de $\mathrm{pH} 7$ (domaine de titration des (OH énoliques de la guanine et de l'uracile).

(*) Cette électrophorèse a été réalisée dans les conditions expérimentales suivantes :

pH $7:$ tampon $\mathrm{H}_{3} \mathrm{PO}_{4}-\mathrm{Na} O H: \mu, 0,05: \mathrm{NaCl}: \mu: 0,15$.

'Temps de dialyse : 48 heures.

Température : $0,3^{\circ} \mathrm{C}$.

Dissipation calorifique : 0,094 watt $/ \mathrm{cm}^{3}$.

Gradient de potentiel : $\mathbf{3 , 0 6}$ volts $\mathrm{cm}^{-1}$.

Temps d'électrophorèse : 35 minutes. 


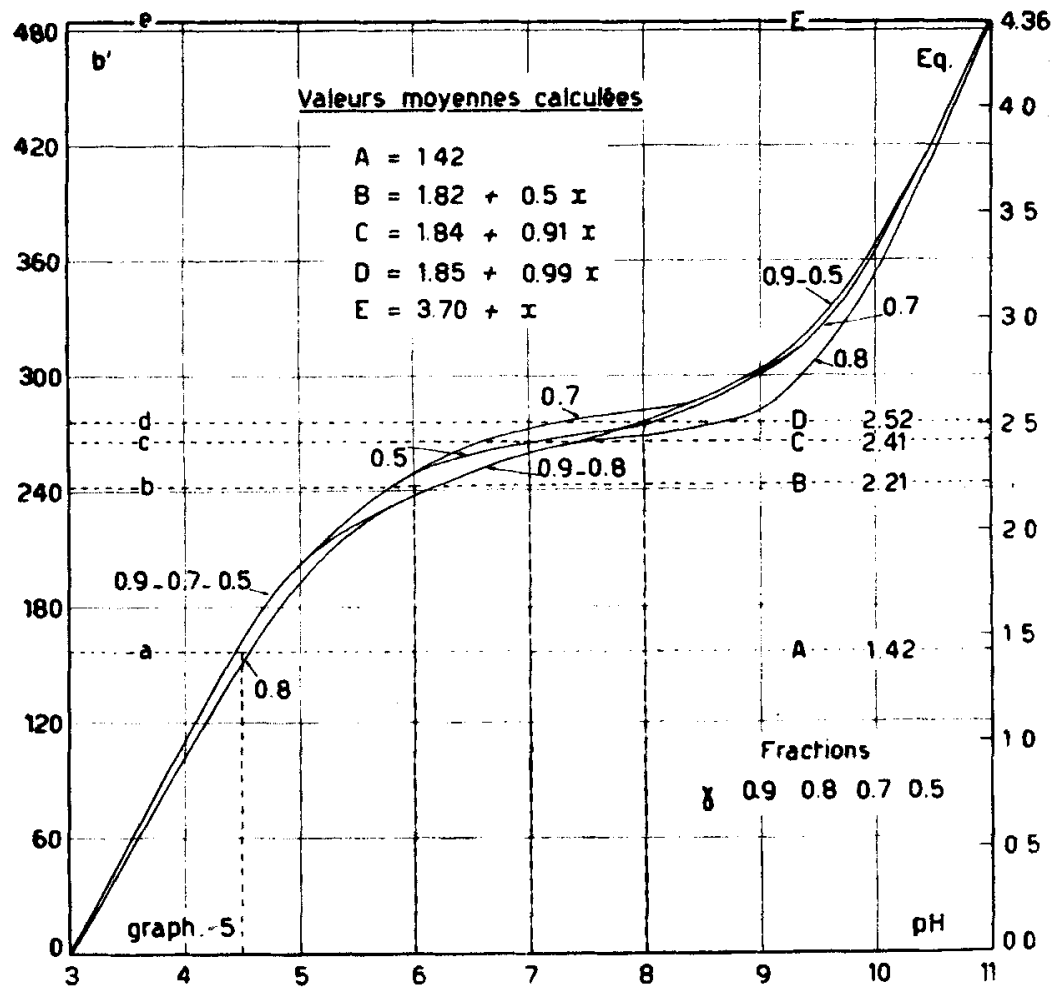

Dans l'ensemble cependant, les différentes courbes obtenues ont même allure ce qui est conforme aux résultats analytiques chromatographiques.

e) Nombre de groupes $\mathrm{OH}$ phosphoriques secondaires

En acceptant que nous ne titrons dans les limites de pH 3-4,5 que les groupes $\mathrm{NH}_{2}$ de la guanine, de l'adénine et de la cytosine, caractérisés par les $\mathrm{pK}$ mentionnés dans le tableau IV, nous pouvons calculer le nombre de fonctions $\mathrm{OH}$ phosphoriques secondaires (de pK supposé égal à 6,0), la somme des bases puriques et pyrimidiques étant à nouveau posée égale à quatre.

Soit (a) la quantité moyenne de base fixée entre $\mathrm{pH} 3$ et 4,5 par cette quantité arbitraire d'acide nucléique, et égale respectivement à 162 et $158 \cdot 10^{-6}$ équivalents pour les fractions relatives aux graphiques 4 et 5 . 
Connaissant d'autre part la composition chimique de nos fractions, nous pouvons calculer le nombre d'équivalents de potasse (A) que fixent théoriquement les bases de l'acide nucléique entre les mêmes limites de $\mathrm{pH}$, soit :

$A=0,16$ [guanine] $+0,70$ [adénine] $+0,61$ [cytosine].

$=1,44$ (fraction $\gamma 0,4 \rightarrow 0,0)$ (valeur moyenne)

$=1,42$ ( (raction $\gamma 0,9 \rightarrow 0,5$ ) (valeur moyenne).

Le nombre de groupes $\mathrm{OH}$ phosphoriques secondaires peut alors ètre trouvé en traçant sur les graphiques 4 et 5 une deuxième échelle des ordonnées exprimée en équivalents de potasse fixés par les quatre môles de bases puriques et pyrimidiques, de manière à amener en coincidence les valeurs (a) et ( $\mathrm{A}$ ) des deux échelles ainsi que l'origine zéro.

D'autre part, de la quantité de phosphore minéralisable déterminée dans chaque solution soumise à l'électrotitration et de la valeur (a), nous pouvons trouver une valeur de (A) qui doit coincider avec celle calculée à partir des données chromatographiques, si le pourcentage de phosphore trouvé est correct. Pour toutes les fractions obtenues par extraction fractionnée, la valeur moyenne de $(\Lambda)$ ainsi trouvée est de 1,45 alors que la valeur calculée est de 1,43. L'accord est donc très bon malgré les écarts individuels de $\pm 0,03\left(^{*}\right)$ et la quantité de potasse fixée entre les $\mathrm{pH} 3$ et 4,5 peut donc servir de base pour les calculs ultérieurs.

Le nombre d'équivalents de potasse à fixer théoriquement depuis le $\mathrm{pH} 3$ jusqu'au $\mathrm{pH}$ de $6,7,8$ et 11, est donné par les relations suivantes, où $x$ est le nombre de groupes $\mathrm{OH}$ phosphoriques secondaires, toujours pour quatre môles de base. pH $6: \mathrm{B} \ldots 0,17$ [guanine] $+0,83$ [adénine] - 0,92 [cytosine] $+0,50[x]$

pH $7: \mathrm{C}=0,17$ |guanine] + 0,84 [adénine] $+0,94$ [cytosine] $+0,91|x|$

$\mathrm{pH} 8: \mathrm{D} \quad 0,17$ [guanine] $+0,84$ ladéninel $+0,94$ [cytosine $]+0,99[x]$

pH 11: E $: 1,06$ Iguanine] $+0,81$ [adénine] + 0,86 [uracile $+0,94[$ cytosine $]+|x|$.

l.es valeurs de $x$ peuvent alors ètre déduites directement

(*) Exception faite pour la fraction $\gamma: 0,3$. 
des valeurs théoriques et expérimentales de $B, C$ et $D$ et sont données dans le tableau suivant (VI).

TABLEAU VI

\begin{tabular}{l|cccc}
\hline \multirow{2}{*}{ Fraction } & \multicolumn{4}{c}{ Zône de pH } \\
& $3-6$ & $3-7$ & $3-8$ & $3-11$ \\
\hline$\gamma 0,9 \rightarrow 0,4$ & 0,78 & 0,63 & 0,68 & 0,66 \\
$\gamma 0,4 \rightarrow 0,0$ & 0,70 & 0,61 & 0,70 & 0,65 \\
0,0 & 0,84 & 0,67 & 0,75 & 0,65
\end{tabular}

$\mathrm{Vu}$ la précision des mesures et l'incertitude des pK, nous pouvons considérer ces valeurs comme assez concordantes.

Il est évidemment toujours possible d'obtenir un meilleur accord en modifiant légèrement les valeurs de certains $\mathrm{pK}$ mais ces corrections ont un caractère assez arbitraire. Puisque entre $\mathrm{pH} 6$ et 8 , nous ne titrons pratiquement que les groupes $\mathrm{OH}$ secondaires de l'acide phosphorique, il est assez logique d'essayer d'obtenir une meilleure concordance en donnant au $\mathrm{pK}$ moyen de ces groupes une valeur légèrement différente de 6,0, valeur que l'on calcule aisément à partir des données des graphiques 4 et 5 et de la relation :

$$
\mathrm{pH}=-\mathrm{pK}+\lg \frac{\alpha}{1-\alpha}
$$

En adoptant un pK de 5,8 , nous trouvons ainsi pour $x$ les valeurs suivantes (tableau VII).

TABLWAL VII

\begin{tabular}{c|cccc}
\hline \multirow{2}{*}{ fractions } & \multicolumn{4}{c}{ zone de $\mathrm{pH}$} \\
& $3-6$ & $3-\tau$ & $3-8$ & $3-11$ \\
\hline$\gamma 0,9 \rightarrow 0,4$ & 0,64 & 0,61 & 0,67 & 0,60 \\
$0,4 \rightarrow 0,0$ & 0,57 & 0,60 & 0,69 & 0,65 \\
0,0 & 0,69 & 0,66 & 0,74 & 0,65
\end{tabular}

(les coefficients $\alpha$ ont pour valeur respective à $\mathrm{pH} 6,7$ et $8: 0,61,0,93,0,99$ )

Nous pouvons donc admettre comme assez plausible que, par quatre bases puriques et pyrimidiques de l'acide nucléique, 
il y a, en moyenne, 0,65 groupes $\mathrm{OH}$ phosphoriques secondaires de $\mathrm{pK}$ approximatif de 5,8 .

La concordance satisfaisante des valeurs de $x$ ainsi trouvées, montre qu'une courbe électrotitrimétrique calculée à partir des données chromatographiques, des pK adoptées et de $x$, doit coïncider avec la courbe expérimentale. Il en est bien ainsi comme le prouve le graphique 6 , sauf aux environs de $\mathrm{pH} 9$ où il $\mathrm{y}$ a légère divergence (la courbe $\gamma 0,9 \ldots-0,5$ est décalée par rapport à la courbe $\gamma^{0,4} 0,0$ de 0,6 équivalents).

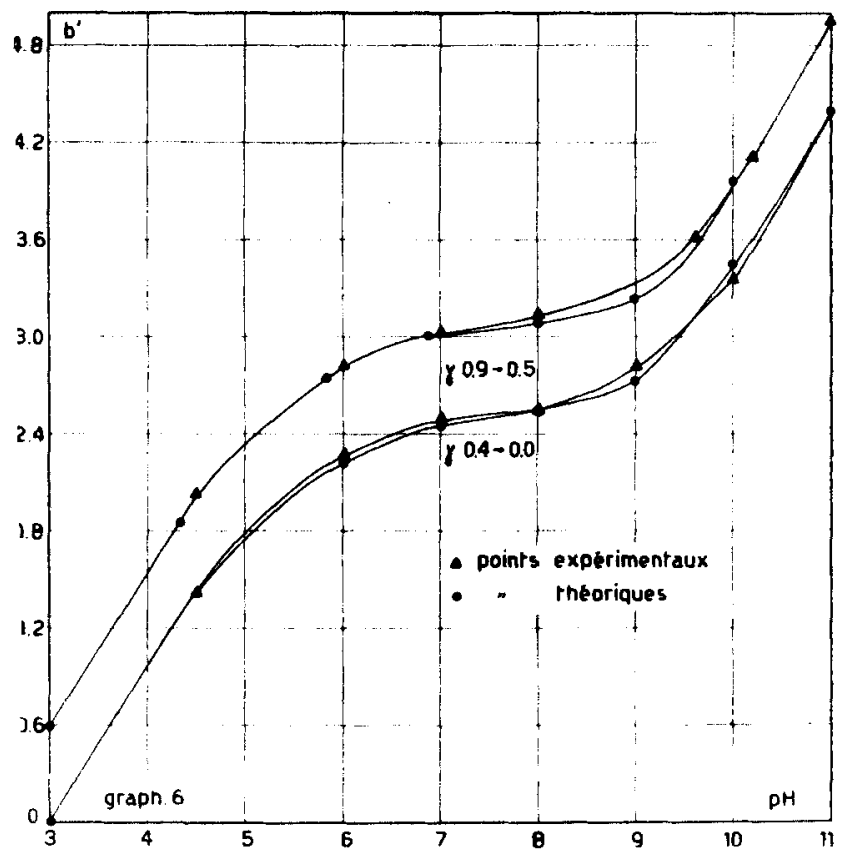

Pour chaque fraction individuelle, il est possible de faire coïncider la courbe expérimentale avec une courbe calculée en assumant un nombre de groupes $\mathrm{OH}$ phosphoriques secondaires légèrement différent dans chaque cas ainsi qu'un pK de valeur proche de 5,8. Nous croyons cependant que ces calculs n'ont pas beaucoup d'intérèt aussi longtemps que l'on n'est pas assuré de pouvoir disposer d'échantillons suffisamment homogènes, bien que de poids moléculaires différents.

L.es courbes d'électrotitration des fractions $\mathrm{A}, \mathrm{A}^{\prime}{ }_{1}$ et $\mathrm{B}$ 
sont données dans le graphique 7 . Nous rappelons que les fractions $\mathrm{A}$ et $\mathrm{B}$ ont été obtenues par emploi du précipitant acide de Chantrenne qui entraîne une légère dégradation du

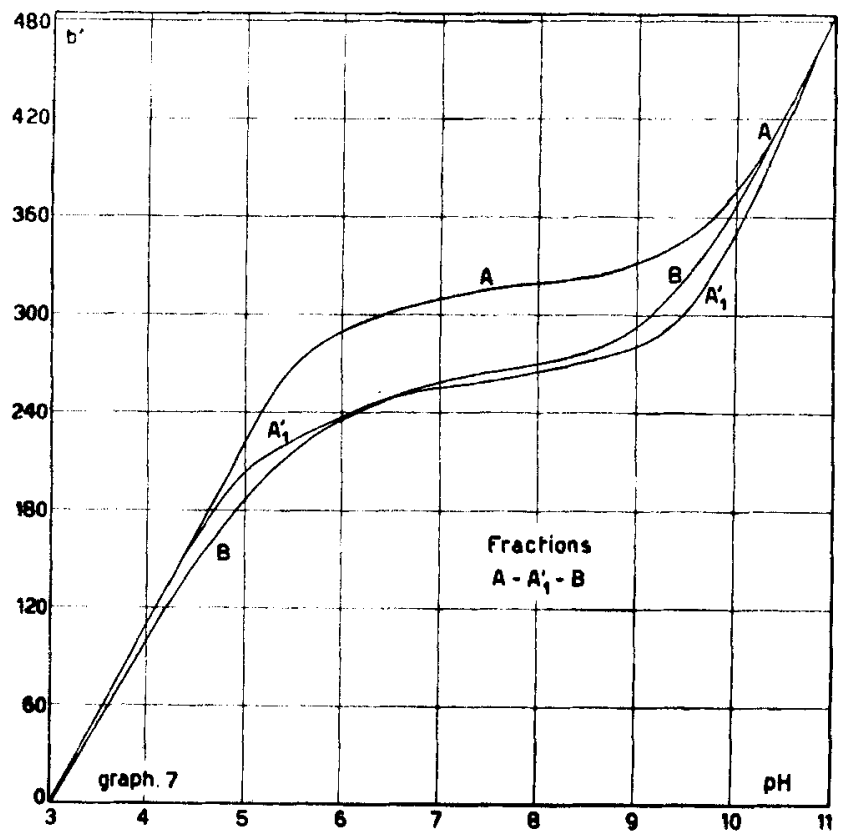

produit $\mathrm{A}$ au cours du fractionnement répété, ce que révèle également la courbe d'électrotitration de cette fraction.

En effet si les courbes du $B$ et $d u A^{\prime}{ }_{1}$ sont normales, en ce sens que leur allure est identique à celle des courbes des fractions obtenues par extraction fractionnée, compte tenu de leur composition chimique différente en bases puriques et pyrimidiques, il n'en est pas de même pour la fraction A.

En utilisant les valeurs de $\mathrm{pK}$ adoptées précédemment, nous trouvons que le nombre de groupes $\mathrm{OH}$ phosphoriques secondaires est de 0,53 pour la fraction $\mathrm{B}$ ( $\mathrm{pH} 6: 0,50$; pH $7: 0,55 ; \mathrm{pH} 8: 0,55 ; \mathrm{pH} 11: 0,57)$ et pour la fraction $\mathrm{A}_{\mathbf{1}}$ (pH $6: 0,65 ; \mathrm{pH} 7: 0,55 ; \mathrm{pH} 8: 0,40 ; \mathrm{pH} 11: 0,50)$.

Par contre, pour la fraction $\mathrm{A}$, les valeurs de $x$ sont très divergentes $(\mathrm{pH} 6: 1,20 ; \mathrm{pH} 7: 0,93 ; \mathrm{pH} 8: 0,97 ; \mathrm{pH} 11$ : 0,70 ). En supposant le $\mathrm{pK}$ moyen du groupe phosphorique secondaire être de 5,45, on trouverait pour $x$ au $\mathrm{pH}$ de 6,7 et 8 les valeurs suivantes : 0,$94 ; 0,92 ; 0,96$; en supposant 
en outre que le $\mathrm{pK}$ moyen des groupes $\mathrm{OH}$ énoliques de la guanine et de l'uracile, est légèrement plus élevé $(10,50)$, on trouverait au $\mathrm{pH} 11$ une valeur de $x$ égale à 0,95 en bon accord avec les trois valeurs précédentes.

\section{IV. - Conclusions générales}

a) Pour toutes les fractions sauf pour la fraction $\mathrm{A}$ qui a été soumise pendant un temps assez long à un pH acide, les données chromatographiques et électrotitrimétriques concordent relativement bien, moyennant une légère correction de certains $p K$. Pour cetle fraction anormale $A$, il faut admettre un nombre de groupes $\mathrm{OH}$ phosphoriques secondaires plus grand, et un pK des OH énoliques plus élevé. $\Lambda$ une charge plus élevée du groupe phosphate correspondrait donc un caractère moins acide du groupe - $\mathrm{NH}-\mathrm{CO}-$ ce qui est conforme a la théorie de Bjerrum ( ${ }^{11}$ ) et de Kirkwood et Westheimer $\left({ }^{12}\right)$.

b) Comme nous l'avons signalé plus haut dans le cas des données chromatographiques, nous croyons qu'il est également dangereux de tirer des courbes d'électrotitration des conclusions trop hâtives sur la structure de l'acide ribonucléique de la levure, cette macromolécule étant manifestement polydispersée. Il est cependant assez étrange de constater que le nombre de groupes $\mathrm{OH}$ phosphoriques secondaires dans toutes les fractions (sauf la fraction $\Lambda$ ) soit relativement constant, environ un groupe pour six bases puriques et pyrimidiques.

Le poids moléculaire moyen du résidu tétranucléotidique de composition conforme à nos données chromatographiques est d'environ 1300; or, les poids moléculaires moyens de nos fractions analysées par électrotitrimétrie varient de 4000 à 38.000 ; on devrait donc s'attendre à observer pour les fractions de très bas poids moléculaires une augmentation du nombre de ces groupes $\mathrm{OH}$ phosphoriques titrables. Il n'en est rien; nous sommes donc amenés à interpréter ce fait en

(11) N. Buenrum : Z. phys. Chem., 106-219 (1923).

$\left.{ }^{12}\right)$ J.G. Krrkwood and F.H. Westheimer : I. Chem. Phys., 6, 506 (1938); 6, $513(1938)$. 
nous basant sur les résultats récents de Markham et Smith ${ }^{(3)}$ et à supposer provisoirement que la formation de produits de dégradation de bas poids moléculaires ne s'accompagne pas de la libération de groupes $\mathrm{OH}$ phosphoriques secondaires libres; ceux-ci formeraient un anhydride cyclique par estérification avec le ribose en position $2^{\prime}$ ou 3 '.

c) Nous avons vu précédemment que la dépolymérisation observée à $\mathrm{pH} 3$ n'entraîne également aucune libération apparente de ces groupes puisque les courbes d'électrotitration ont mème allure avant et après ce traitement. Si cette dépolymérisation se fait avec libération des groupes $\mathrm{OH}$ phosphoriques primaires par rupture de liaison R-O-P du

groupe R-O-P-(OR) $)_{2}$, il serait évidemment impossible de détecter dans les courbes d'électrotitration cette transformation puisque nos courbes sont limitées au domaine de $\mathrm{pH}$ de 3 à 11.

Si la dépolymérisation entraîne la rupture d'une liaison avec libération d'un groupe $\mathrm{OH}$ phosphorique secondaire, il faudrait admettre que cette hydrolyse se ferait également avec formation d'anhydride cyclique. Il n'est pas exclu cependant que la dépolymérisation de l'acide ribonucléique de poids moléculaire élevé ne soit due à la rupture de liaisons secondaires $\left({ }^{14}\right)$; rien ne milite cependant sur la base de nos connaissances actuelles en faveur de l'une ou l'autre de ces hypothèses.

d) L'impossibilité de détecter une hétérogénéité par électrophorèse dans le cas de l'acide ribonucléique de la levure $\left.{ }^{9}\right)$ prouve que la dégradation doit se faire sans modification importante de la charge. C'est ce que prouvent également les courbes d'électrotitration.

e) La dépolymérisation de l'acide nucléique donne naissance à un système polydispersé constitué de molécules de composition chimique relativement constante; il s'en suit que, non seulement les bases elles-mêmes doivent être réparties plus ou moins statistiquement dans toute la molécule

(13) R. Markham and J.D. Smith : Nature, 168, 406 (1951).

(14) R. Thomas : Experientia, VII, 17, 261, 1951. 
mais que la distribution des chaînes latérales fixées sur la chaîne principale présente une certaine régularité.

Si nous adoptons l'hypothèse d'une chaîne principale formée essentiellement de bases puriques sur laquelle sont greffées des chaînes latérales de bases pyrimidiques, nous devons également admettre une répartition statistique de ces chaînes le long de l'axe de la molécule. Il serait cependant nécessaire d'avoir plus de précisions sur les caractéristiques moléculaires du résidu de l'action de la ribonucléase car, si ce résidu est de faible poids moléculaire, il n'est pas exclu qu'il soit simplement une partie de la molécule plus riche en bases puriques suite à une élimination préférentielle des bases pyrimidiques et qu'il n'ait par conséquent que peu de signification structurale.

Cette étude a été subsidiée par le Centre National de Biologie Physico-chimique, par le Centre de Recherches pour la Pénicilline et les autres Antibiotiques et par la Fondation Rockefeller.

Nous tenons à remercier ces organismes pour leur appui, ainsi que l'I.R.S.I.A. pour l'octroi d'une bourse de spécialisation à l'un de nous (J. G.).

UNIVERSITÉ DE LIIÈGE

Laboratoire de Chimie physique. 